\title{
Using Static Parametric Design to Support Systems Engineering of Industrial Automation Systems
}

\author{
Hongchao $\mathrm{Ji}^{1} \quad$ Lars Mikelsons $^{1} \quad$ Karl Kempf $^{1} \quad$ Dieter Schramm $^{2}$ \\ ${ }^{1}$ Bosch Rexroth AG, Lohr am Main, Germany \\ \{hongchao.ji, lars.mikelsons, karl.kempf\}@ boschrexroth.de \\ ${ }^{2}$ University of Duisburg-Essen, Duisburg, Germany \\ dieter.schramm@uni-due.de
}

\begin{abstract}
This paper proposes a static parametric design methodology for application of the model based systems engineering (MBSE) paradigm in the world of Modelica. This methodology allows for parameter synthesis of the industrial automation systems under consideration of customer requirements. Furthermore, the parametrized system can be verified automatically. An integrated system model consisting of requirements, system design and verification models is created and can be used as a design template to generate a new parameter set according to the change of customer requirements. A case study from the practice is presented to proof the concept of this methodology.
\end{abstract}

Keywords: Model Based Systems Engineering, SysML, Modelica, Parameter Synthesis

\section{Introduction}

The complexity of modern industrial automation systems increases steadily. New functions and technologies need to be integrated to fulfill customer requirements, environmental regulations and/or safety standards. The increasing complexity has raised many challenges such as keeping the the design consistent and approving the correctness with respect to the customer requirements. Model based systems engineering (MBSE) is defined as the formalized application of modeling to support system requirements, design, analysis, verification and validation activities beginning in the conceptual design phase and continuing throughout development and later life cycle phases. Hence MBSE is a suitable approach to cope with these challenges.

One of the key issue in MBSE process is to determine the proper dimension of the system design ac- cording to the formalized requirements model. The static parametric design methodology uses Modelica static models together with the dynamic models to support the MBSE process by the means of selecting the proper components of the desired system from given product catalogs, dimensioning the sub-systems as well as checking the correctness of the system design with respect to systems requirements.

The objective of the static parametric design methodology is to perform a parameter synthesis of a technical system according to the customer requirements automatically. Furthermore, the calculated system design can be verified automatically as well. The Systems Modelling Language (SysML) [11] is used to formalize the customer requirements. Moreover, the extension of abstraction levels and classification defined in [4] is also applied in this paper. Work on the integration of SysML and Modelica has already proven its effectiveness in the MBSE $[6,8,9]$. Reusing these improvements the SysML models can be transformed into executable Modelica models.

In this contribution, we focus on the standard application that the structure of the desired system is normally known to the system engineers. The requirements models serve as the basis of the static parametric design methodology. By changing the requirements models, a new parameter set of the system can be obtained automatically. In this sense, the integrated model consisting of requirements, system design and verification models can be seen as a design template for a standard application.

This paper is organized in 6 sections. Section 2 illustrates the current systems engineering process and the need of model based systems engineering and static parametric design methodology. As part of related work in Section 3 a short introduction to SysML and its integration with Modelica are given. Section 4 introduces the static parametric design methodology 
to support the systems engineering in detail. The capabilities of the proposed methodology are demonstrated using an industrial application in Section 5. The paper closes with conclusions and an outlook to future work.

\section{Systems Engineering of Industrial Automation Systems}

The systems engineering process is described in the following referring to the well known V Model according to the VDI 2206 standard [12] depicted in (Figure 1).

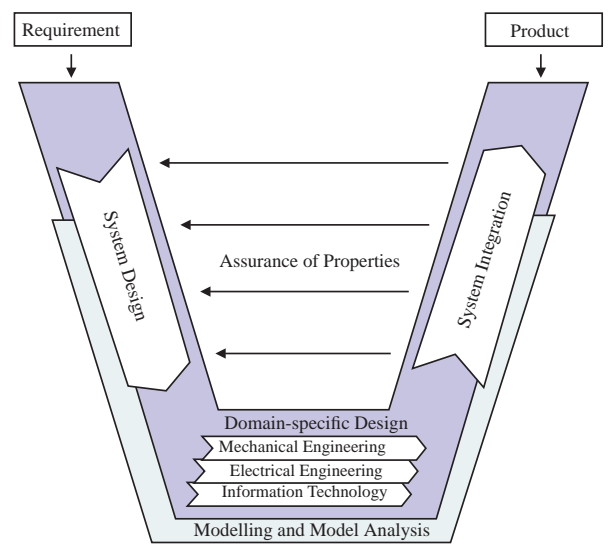

Figure 1: V Model According to VDI 2206 [12]

The main tasks of current systems engineering of industrial automation systems can be summarized as follows:

- State the customer needs correctly and unambiguously;

- Define the proper system design based on the customer requirements;

- Verify the system design against customer requirements.

They will be introduced respectively in the following.

\subsection{Requirements Specification}

Due to the fact that the requirement specification is the subject matter of contract between customer and contractor the above described context implies that the requirements engineering has to be seen not only from the technical perspective but also needs to consider the business process and the contractual situation along the supplier chain. In this context it is self-evident that the requirements shall be defined and structured not only according to technical aspects but also according to the contractual situation. The definition of levels of abstraction is an appropriate way to meet these needs. The depicted levels of abstraction in Figure 2 reflect the described supplier chain and major technologies involved and therefore are a reasonable choice in the context of automation systems. In order to deal with the complexity of large systems the design objects are clustered in a system break down structure. The requirements derived on the different levels of abstraction can be referenced in requirement specifications in order to provide the contractual views on subsets of requirements.

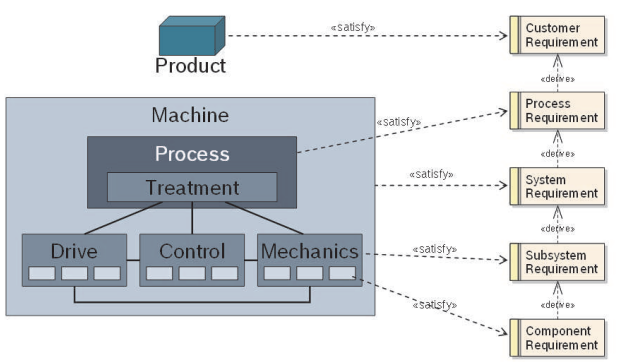

Figure 2: Levels of Abstraction in Requirements and System Design

\subsection{Systems Design}

Industrial automation systems are characterized by their ability to process a material or work piece according to a defined procedure to achieve the output of a desired product. The challenge of the system engineer is to design a machine that is capable to run the process in a deterministic and efficient way. This task is typically performed within a specific design domain that refers to a field of technical expertise. The proper selection of the components and their integration into the overall structure strongly influence the function, performance, robustness and reliability of the whole system. Currently the selection of the components is mainly determined by the competence of the system engineers which is time consuming and error prone. In order to avoid manual errors, it is desired to select the components in a systematical manner.

Today, parameter synthesis of a technical system is usually based on static calculations in the field of industrial automation systems. A small example of such static calculation for selection of a hydraulic valve in a hydraulic lift system is depicted in Figure 3. The dimension of the cylinder shall be first defined in order to calculate the maximal flow rate through the valve 
with the given maximal cylinder velocity by

$$
Q_{\max }=A_{D} \cdot v_{\max } .
$$

After that the nominal size of the valve is determined by some design criteria. Moreover, the design criteria origin from customer requirements as well. In this case, the nominal size is defined by the nominal flow rate which is calculated by

$$
Q_{\text {nominal }}=1.5 \cdot Q_{\max }
$$

The nominal size of the valve can be chosen from the product catalog according to the nominal flow rate. That means a proper component, in this case the valve, is correctly selected for the desired system.

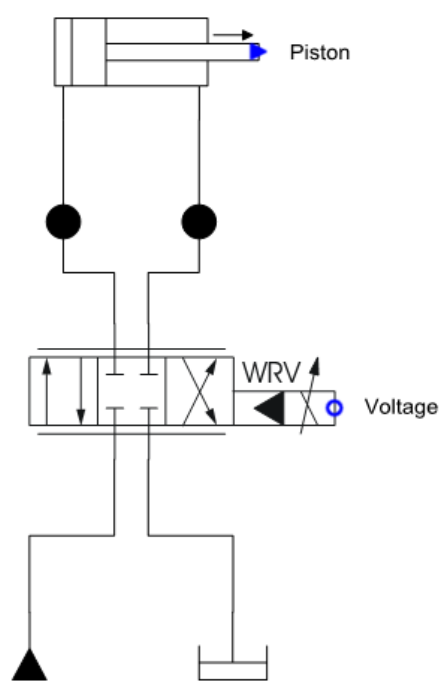

Figure 3: Schematic of a Hydraulic Lift System

In order to ensure the acceptance of a MBSE tool offering the methodology presented in this paper, these design guidelines have to be integrated into the MBSE tool.

\subsection{Verification and Validation}

Model based verification and validation of a systems design against systems requirements has been widely used in the field of industrial automation systems. The goal here is to verify the system design in an automated and reproducible way. Since this issue has already been addressed in virtual verification of systems design against systems requirements (vVDR) methodology [10], it is used to approve the systems design in this paper as well.

\subsection{The Challenges}

Since industrial automation systems usually consist of components from different domains, it is hard to keep the design correct and consistent. In order to deal with this fact, the systems design from different engineering domains shall be integrated into the whole MBSE process. Therefore, a universal and standardized modeling language is required which shares the understanding among engineers from different disciplines. This common language shall enable the generation of requirements models, system design models, traceability models as well as verification models containing domain-specific details. SysML is being proposed to meet this requirement. However it has been evaluated as not sufficient due to the lack of executable semantics. Integration of the languages SysML and Modelica has proven its efficiency in the area of MBSE $[5,6,7,10]$. Therefore, in this contribution SysML and Modelica are chosen as the modelling languages applied in our MBSE process as well.

In order to set up a MBSE tool for parameter synthesis, the following two questions have to answered:

1. How to perform a parameter synthesis to determine all the proper components based on the customer requirements in an automatic manner?

2. How to link different kinds of models in the whole MBSE process?

These two challenges have been addressed in this paper by the means of using static parametric design methodology in an integrated system model based on SysML and Modelica. The details are presented in Section 4.

\section{Background and Related Work}

\subsection{The SysML and Modelica}

SysML is a general purpose language used in the field of systems engineering. It is defined as a UML profile which reuses subsets of UML constructs and extends them with some additional modeling elements. SysML is capable to capture the textual requirements and to allocate them with the design models and test cases. However due to loosely defined executable semantics SysML is not capable to execute the modeled physical systems. In contrast to that, Modelica is an object oriented and equation based modeling language for multi-domain physical systems. Graphical modeling is supported by the object diagram which offers an 
intuitive way to describe power transmission through acausal connections as well as directed signal flows. Strong semantics allow the generation of executable models of continuous as well as discrete systems. Object oriented language constructs enable the efficient reuse of models and the design of comprehensive and easy to use model libraries. As mentioned in Section 2, a language which integrates the descriptive modeling power of SysML and the formal executable simulation power of Modelica seems to be a promising approach for the systems engineering in industrial automation systems.

\subsection{Related Work}

Several work has already been done towards application of MBSE paradigm using Modelica language with different concerns. The vVDR methodology [10] addresses mainly the virtual verification of systems requirements by using UML, Modelica and ModelicaML. In Dubois et. al. [2] a requirement traceability model to enforce the traceability concept in SysML in the automotive domain is presented. Requirements management and allocation have already been covered in the other paper of the author [4].

This paper describes a methodology for the parameter synthesis of technical systems according to customer requirements. Moreover, the different kinds of models are linked with the other models in the integrated system model and therefore it is easy to regenerate and to verify the final parametrized system. They will be introduced in detail in the following section.

\section{Static Parametric Design}

The proposed static parametric design methodology is based on Modelica static models. Static models are defined as models that are constant over time. Considering the whole MBSE process, the following items can be formalized as Modelica static models:

- Requirement Specifications,

- Product Catalogs,

- Selection Criteria.

\subsection{Definitions of Models}

The requirement specifications can be defined as requirements models which are captured as stereotyped SysML models according to the different abstraction levels and classifications in [4].
This classification is mainly based on the taxonomy proposed in [3] with some changes as presented in the following. Instead of the requirement type specific quality, the structural requirement is defined in the field of industrial automation.

- A functional requirement is the requirement that should produce an expected reaction to a given stimuli.

- A performance is the requirement to check whether a system variable such as timing, speed, volume or throughput is in a desired range.

- A structural requirement is the requirement which describes the structural demand of the stakeholder.

- A constraint is the requirement to provide the technical and safety boundary conditions that the system shall satisfy.

The product catalogs models are easy to understand as Modelica static models. They can modeled as a simple record class with some table definitions. In this work, a UML library with a sub-set of the Bosch Rexroth catalog is implemented and later transformed to Modelica static models. The advantages of implementation as UML library over Modelica library is the compatibility with SysML and the extendibility of the product catalog.

The selection criteria are implemented as static calculation models. The idea is using Modelica functions to determine the proper dimension of the components. Normally, those selection criteria for the components are usually the same. Due to the fact of reuseability, a Modelica library called ParametricDesign which consists of most selection criteria in the field of industrial automation systems is built as shown in Figure 4.

Besides the static models, simulation model is an executable model which is used for dynamic simulation. In the verification and validation phase, the simulation model is linked with the test cases to check the correctness of the parametrized system design.

All those models are further transformed into Modelica static models with the help of a Modelica code generator, which is implemented with the help of Eclipse Acceleo [1].

\subsection{Link of Different Models}

Several models have been defined in this static parametric design methodology. It is necessary to link 


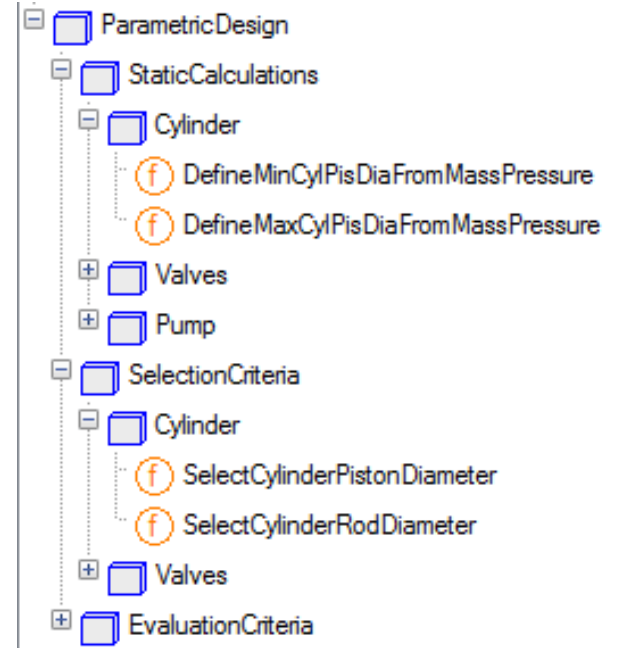

Figure 4: Structure of the Parametric Design Library

those models in a efficient manner in order to perform a parameter synthesis automatically. The basic idea is to reference the attributes of SysML model to the variables, parameters and constants of the Modelica model. Currently, these relations have been established manually which is time consuming and error prone. A method to extend the standard relationships such as «satisfy», «verify» and «derive» has been proposed in [4]. An overview of the linking of different models in this methodology is shown in Figure 5. The tooling that supports the binding of related objects is implemented in Eclipse.

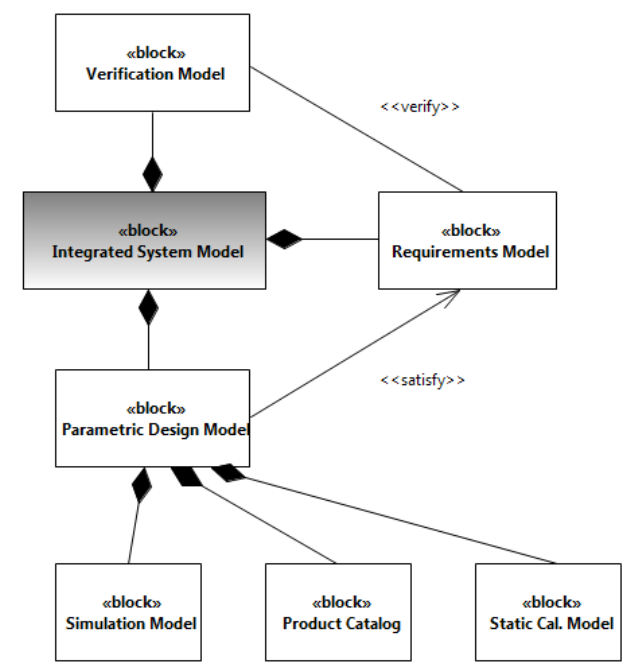

Figure 5: Link of Different Models

\subsection{Methodology Description}

The prerequests of application of the static design methodology are

- hydraulic library,

- static design library,

- product catalog library.

Furthermore, the formalized requirements models and at least one simulation model have to be created at first as the basis for application of the static parametric design methodology.

The main steps of this methodology can be summarized as follows:

1. Capture the customer requirements as stereotyped requirements according to the proposed classification in [4].

2. Create a simulation model from the hydraulic library on the considered level of abstraction.

3. Select the proper design criteria from the parametric design library and create the static calculation model.

4. Link the requirements model, static calculation model as well as product catalog model in the parametric design model.

5. Run a parameter synthesis to obtain a best suited parameter set and the other possible parameter sets for the desired system.

6. Set the obtained possible parameter sets in the simulation model and save them as design variants.

7. Define test cases that need to satisfy customer requirements.

8. Link the requirements model, test cases as well as simulation models in the verification model.

9. Run a verification that executes all related test cases and design variants.

10. Choose the best suited design variants according to the verification results. 


\section{Application Example}

In this section, a hydraulic lift system is used to demonstrate the static parametric design methodology. The hydraulic lift system is used to lift a load to a given height. It shall be considered in the context of the OEM-supplier relation as it applies to a typical Bosch Rexroth engineering project.

The task of this case study is to define a best suited parameter set of the desired lift system which fulfills all the customers requirements as well as technical constraints. First of all, a simulation model shall be created. Therefore, the structure of the hydraulic lift system has to be known for the system engineers. Then, the static calculation model shall be created as well by selecting the proper design criteria from the parametric design library. After linking of different models in the integrated system model, a parameter synthesis can be performed to obtain the best combination of the components with the minimal dimension which satisfy all the requirements.

The main advantage is that the system engineers can use the integrated system model as a design template. With the help of this design template, it is much more easier to variate the parameter set of the hydraulic lift system by changing the customer requirements automatically.

\subsection{Requirements Capture}

The requirements from the customers are formalized as follows: a load of $3000 \mathrm{~kg}$ shall be lifted to $0.5 \mathrm{~m}$ within $2 s$. Besides the customer requirement there are some technical constraints of the desired system. For example, the maximum velocity can not exceed 0.6 $\mathrm{m} / \mathrm{s}$. The other important constraint is that the pressure drop over the proportional valve shall not exceed $30 \%$ of the working pressure. The important requirements are listed in Table 1. The beginning letter of the ID of the requirement refers to the type of the requirement. The requirements $\mathrm{P} 1, \mathrm{C} 2$ and $\mathrm{C} 3$ can be verified by test cases which are modeled as Modelica models. Since the structral requirments S4 and S5 provide only the important design parameters, they are not necessary or possible to verify. According to those requirements, the proper components from the product catalogs shall be selected iteratively until all the components are chosen. They can be formalized as SysML requirements model and later transformed into Modelica static model. An example of the requirements model and its generated Modelica code are shown in Figure 6.

\begin{tabular}{ll}
\hline ID & Description \\
\hline P1 & The load shall be lifted to $0.5 \mathrm{~m}$ within $2 \mathrm{~s}$. \\
\hline C2 & The max. velocity shall not exceed $0.6 \mathrm{~m} / \mathrm{s}$. \\
\hline C3 & $\begin{array}{l}\text { The pressure loss over the valve shall not } \\
\text { exceed } 30 \% \text { of the working pressure. }\end{array}$ \\
\hline S4 & The mass of the load is $3000 \mathrm{~kg}$. \\
\hline S5 & The working pressure is $200 \mathrm{bar}$. \\
\hline
\end{tabular}

Table 1: The System Requirements List

\begin{tabular}{|l|}
\hline \multicolumn{1}{|c|}{$\begin{array}{c}\text { wrequirement } \\
\text { DesiredHeight }\end{array}$} \\
\hline $\begin{array}{l}\text { sRequirement } \\
\text { id=001 } \\
\text { text=A load of } 3000 \mathrm{~kg} \text { shall be lifted to } 0.5 \mathrm{~m} \text { within } 2 \mathrm{~s}\end{array}$ \\
\hline $\begin{array}{l}\text { + actPos: ModelicaReal } \\
\text { + desPos: ModelicaReal } \\
\text { + diffPos: ModelicaReal } \\
\text { + eAllowed : ModelicaReal } \\
\text { + violated : ModelicaBoolean } \\
\text { + desTime: ModelicaReal }\end{array}$ \\
\hline
\end{tabular}
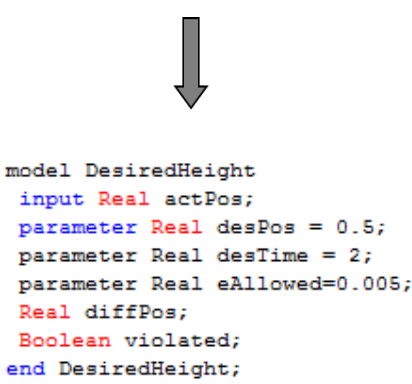

Figure 6: An Example of Requirements Model and Generated Modelica Code

\subsection{Modelling of the Hydraulic Lift System}

The object diagram (Figure 7) shows the structure of the hydraulic lift system. The load is lifted by a differential cylinder which is driven by a constant pressure source. The proportional valve is controlled by a simple P-controller to realize the position control. Since the focus of this work is to illustrate the static parametric design methodology, the details about the model will not be introduced here.

\subsection{Static Parametric Design Process}

The involved components from the product catalog are the differential cylinder and proportional valve. Hence, the design criteria models for those two components in the design library are selected into the static parametric design model together with the requirements model. Table 2 and 3 show the important design variables from the product catalogs of differential cylinder and proportional valve. 


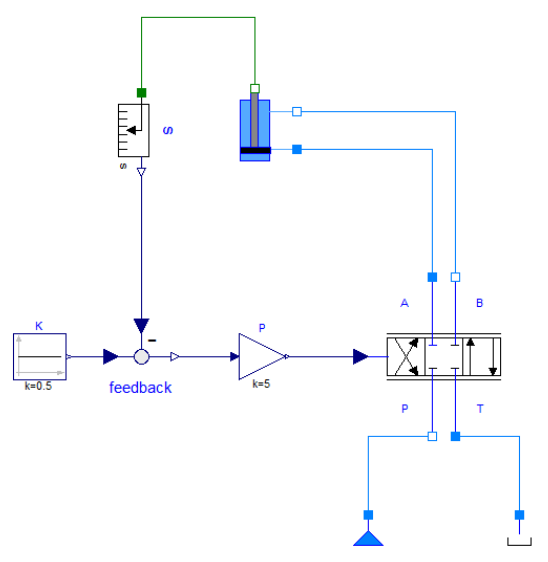

Figure 7: Object Diagram of the Hydraulic Lift System

\begin{tabular}{c|c|c}
\hline $\begin{array}{c}\text { Piston } \\
\text { Diameter } \\
\mathrm{mm}\end{array}$ & $\begin{array}{c}\text { Rod } \\
\text { Diameter } \\
\mathrm{mm}\end{array}$ & $\begin{array}{c}\text { Max } \\
\text { Stroke } \\
\mathrm{mm}\end{array}$ \\
\hline 40 & 28 & 2000 \\
\hline 50 & 36 & 2000 \\
\hline 63 & 45 & 2000 \\
\hline 80 & 56 & 2000 \\
\hline 100 & 70 & 3000 \\
\hline 125 & 90 & 3000 \\
\hline 140 & 100 & 3000 \\
\hline
\end{tabular}

Table 2: Product Catalog of Differential Cylinders

\begin{tabular}{c|c|c}
\hline $\begin{array}{c}\text { Nominal } \\
\text { Size } \\
-\end{array}$ & $\begin{array}{c}\text { Max } \\
\text { Flow Rate } \\
1 / \text { min }\end{array}$ & $\begin{array}{c}\text { Pressure } \\
\text { Drop } \\
\text { bar }\end{array}$ \\
\hline 10 & 170 & 80 \\
\hline 16 & 450 & 180 \\
\hline 25 & 900 & 350 \\
\hline 27 & 1000 & 430 \\
\hline 35 & 3500 & 1100 \\
\hline
\end{tabular}

Table 3: Product Catalog of Proportional Valves

The requirements variables defined in the requirements models, such as mass of load, maximum velocity and desired lifting position are taken as inputs for the design criteria which are implemented as Modelica functions. Finally, a parametric design model is obtained by linking the requirements models, static calculation models and the related product catalog models.

After the parametric design model is created, the automatic parameter synthesis can be done very conveniently. The parametric design model is interactive solved and the design variables are calculated. Ac- cording to those design variables the corresponding components with the proper size are chosen until all the components from the product catalog are chosen. The following table shows the automatic generated best suite combination of cylinder and valve, which is defined as an optimal design. It is worth to mention

\begin{tabular}{l|c|c|c}
\hline Cylinder & $\begin{array}{c}\text { Piston } \\
\text { Diameter } \\
\mathrm{mm}\end{array}$ & $\begin{array}{c}\text { Rod } \\
\text { Diameter } \\
\mathrm{mm}\end{array}$ & $\begin{array}{c}\text { Max } \\
\text { Stroke } \\
\mathrm{mm}\end{array}$ \\
\hline Optimal & 100 & 70 & 3000 \\
\hline \hline Valve & $\begin{array}{c}\text { Nominal } \\
\text { Size } \\
\text { Optimal }\end{array}$ & $\begin{array}{c}\text { Max } \\
\text { Flow Rate } \\
\text { 1/min }\end{array}$ & $\begin{array}{c}\text { Pressure } \\
\text { Drop } \\
\text { bar }\end{array}$ \\
\hline
\end{tabular}

Table 4: Selected Components from Product Catalog

that the gain of the P-controller is determined by "Try and Error". In the future, this kind of parameter which is not related to the product catalog can be defined by the means of optimization.

\subsection{Verification of System Design}

After the static parametric design process is done, a best suited set of combination of the components is obtained. An automatic verification will check the optimal design against customer requirements. This is done by using vVDR methodology [10] to model the test case of requirements with violation monitor. According to the requirements definitions in Table 1, three test cases are defined to verify the requirements $\mathrm{P} 1, \mathrm{C} 2$ and $\mathrm{C} 3$. Figure 8 shows the verification result of this proposed optimal design. The first two figures (Figure 8(a) and 8(b)) illustrate that the load is lifted to 0.5 meter after 2 second and does not exceed the maximal velocity. As shown in Fiugre 8(c), the pressure loss over the valve in steady state satisfies the critical $30 \%$ of working pressure 200 bar as well. Therefore, the hydraulic lift system with the automatic selected parameter fulfills the customer requirement and technical constraints.

\subsection{Comparison of Design Variants}

The best suited combination of components from the product catalog are supposed to have the minimal size which satisfy all the requirements. It has been verified to fulfill all the requirements in the last section. Nevertheless, it is still not proved that the performance of this design is better than the others variants. In order 


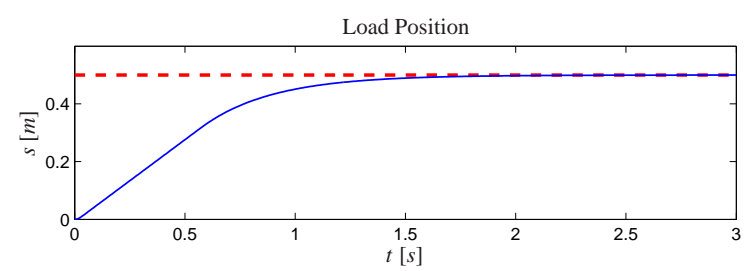

(a) Load Position

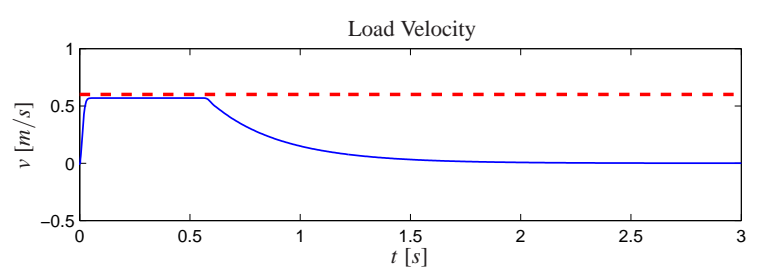

(b) Load Velocity

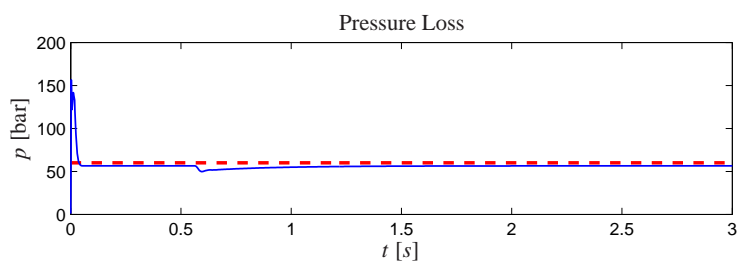

(c) Pressure Loss

Figure 8: Verification Results of Optimal Design

to validate this, design variants around the optimal design with other nominal sizes can be generated from this methodology. The design variants are defined by substituting the components of the optimal design with a smaller or larger nominal size. In this case, four design variants are automatically generated and used to compare with the optimal design. The dimension of all the design variants are shown in the following table.

\begin{tabular}{l|c|c|c}
\hline $\begin{array}{l}\text { Design } \\
\text { Variants }\end{array}$ & $\begin{array}{c}\text { Piston } \\
\text { Diameter }\end{array}$ & $\begin{array}{c}\text { Rod } \\
\text { Diameter }\end{array}$ & $\begin{array}{c}\text { Valve } \\
\text { Size }\end{array}$ \\
\hline Optimal & 100 & 70 & 25 \\
\hline Variant 1 & 80 & 56 & 25 \\
\hline Variant 2 & 100 & 70 & 16 \\
\hline Variant 3 & 100 & 70 & 27 \\
\hline Variant 4 & 125 & 90 & 25 \\
\hline
\end{tabular}

Table 5: Dimensions and Costs of Design Variants

The simulation results of the optimal design and the other four design variants are shown in Figure 9. It shows that the design variant 1 and 3 can approach the desired position within 2 seconds. However the velocities exceed the maximal velocity constraint $0.6 \mathrm{~m} / \mathrm{s}$. The design variant 2 and 4 fulfill the second test case and can not satisfy the first one. The results concerning the third test case are listed in Table 6.

A verification matrix of the design variants against

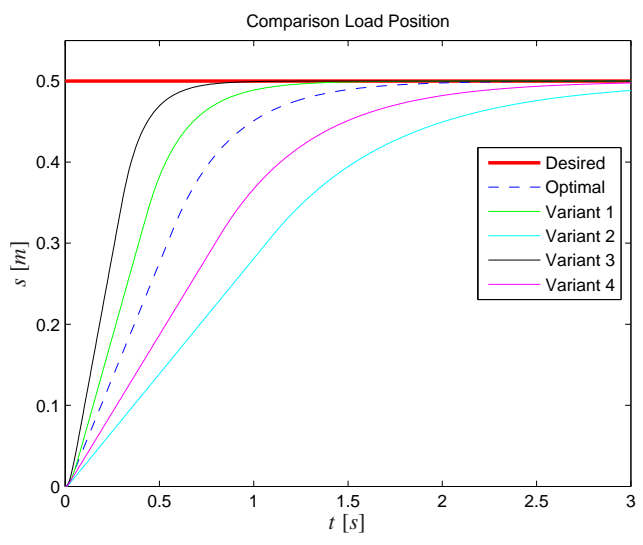

(a) Comparison of Load Position

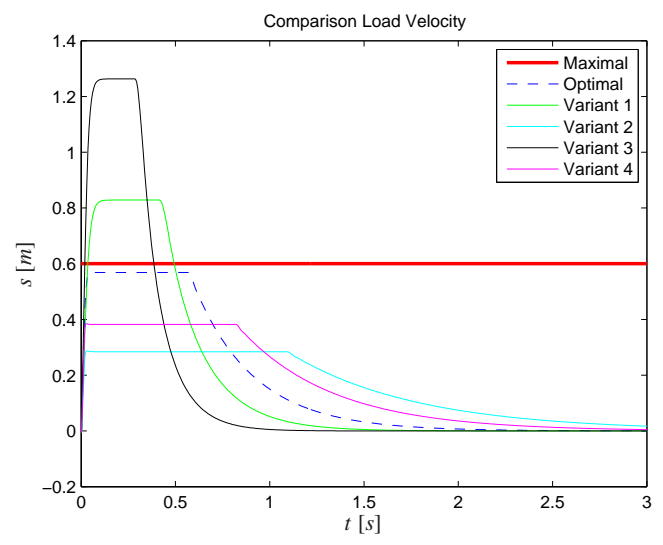

(b) Comparison of Load Velocity

Figure 9: Comparison of Cylinder Positions and Velocities of Different Design Variants

the test cases are shown in Table 6. Furthermore, the costs of design variants depending on the dimensions of selected components from the product catalog can also be calculated. With the help of this verification matrix and the price, the selected optimal combination of the components from the product catalog is proved to be exact the best suited design.

\begin{tabular}{l|c|c|c|c}
\hline Design & Test & Test & Test & System \\
Variants & Case 1 & Case 2 & Case 3 & Cost \\
\hline Optimal & passed & passed & passed & 2000 \\
\hline Variant 1 & passed & failed & passed & 1700 \\
\hline Variant 2 & failed & passed & passed & 1820 \\
\hline Variant 3 & passed & failed & failed & 2040 \\
\hline Variant 4 & failed & passed & failed & 2400 \\
\hline
\end{tabular}

Table 6: Verification Matrix and System Costs 


\subsection{Open Issues}

This case study demonstrates the proposed static parameter design methodology. According to the customer requirements and technical constraints, the dimension of the desired system can be defined automatically. However, the main drawback is that the simulation model shall be first modeled. That means this methodology can not be applied to arbitrary system. This is due to the fact that there is not enough information for determining a proper combination of the desired system in the practice. This drawback also limit the application of MBSE in the field of industrial automation systems.

It is noticed that the order for the selection of components is fixed in this case, i.e., the dimension of cylinder shall be first defined in order to determine the nominal size of the proportional valve. Sometimes the order is not fixed. For both cases the system engineers shall have the chance to determine the order for the selection of components more freely without reimplementation of static design model. Since Modelica is a standardize equation-based modelling language, it has been chosen to meet these requirements. It is capable to deal with the this issue. One proposed concept is to switch the variability of parameter and variable of static models in an arbitrary manner. The system engineers can give the known parameters until the static model is balanced and solvable to calculate the other unknown variables.

\section{Conclusion and Future Work}

In this paper a static parametric design methodology has been analyzed in the systems engineering context of industrial automation systems. A set of possible design variants with different dimensions can be automatically generated and compared by using this methodology. The concept has been demonstrated by a case study of a typical engineering project. The other contribution of this work is allocation of this methodology in a MBSE process in which the parametrized design variants are fully traceable to the other models.

In the future, the proposed methodology will be implemented as an Eclipse plug-in for better tool support of the static parametric design. It is usually the case, not all the parameters can be defined by the static parametric design methodology. Integration of an optimizer to define those parameters is desired. Application of a big scenario is also a part of future work.

\section{Acknowledgments}

This work is funded by Bosch Rexroth AG and German Federal Ministry of Education and Research (BMBF) in the ITEA2 OPENPROD project.

\section{References}

[1] http://www.acceleo.org.

[2] Hubert Dubois, Marie-Agnès Peraldi-Frati, and Fadoi Lakhal. A Model for Requirements Traceability in a Heterogeneous Model-Based Design Process: Application to Automotive Embedded Systems. In 15th IEEE International Conference on Engineering of Complex Computer Systems, ICECCS 2010, Oxford, United Kingdom, 22-26 March 2010, pages 233-242, 2010.

[3] Martin Glinz. On Non-Functional Requirements. In 15th IEEE International Requirements Engineering Conference, RE 2007, October 15-19th, 2007, New Delhi, India, pages 21-26, 2007.

[4] Hongchao Ji, Oliver Lenord, and Dieter Schramm. A Model Driven Approach for Requirements Engineering of Industrial Automation Systems. In Proceedings of the 4th International Workshop on EquationBased Object-Oriented Languages and Tools (EOOLT'07), pages 13-24, 2011.

[5] Thomas Johnson, Christiaan J.J. Paredis, and Roger Burkhart. Integrating Models and Simulations of Continuous Dynamics into SysML. In Proceedings of 6th International Modelica Conference, Bielefeld, Germany, 3-4,March, 2008, 2008.

[6] Christiaan J.J. Paredis, Yves Bernard, Roger M. Burkhart, Hans-Peter de Koning, Sanford Friedenthal, Peter Fritzson, Nicolas F. Rouquette, and Wladimir Schamai. An Overview of the SysML-Modelica Transformation Specification. In 2010 INCOSE International Symposium, July 2010.

[7] Adrian Pop, David Akhvlediani, and Peter Fritzson. Towards Unified System Modeling with the ModelicaML UML Profile. In Proceedings of the 1st International Workshop on EquationBased Object-Oriented Languages and Tools (EOOLT'07), pages 13-24, 2007. 
[8] Wladimir Schamai. Modelica Modeling Language (ModelicaML). Technical report, EADS Innovation Works, Germany, 2009.

[9] Wladimir Schamai, Peter Fritzson, Chris Paredis, and Adrian Pop. Towards Unified System Modeling and Simulation with ModelicaML: Modeling of Executable Behavior Using Graphical Notations. In Proceedings of the 7th International Modelica Conference, Como, Italy, 20-22 September 2009, number 43 in Linköping Electronic Conference Proceedings, pages 612-621. Linköping University Electronic Press, Linköpings universitet, December 2009.

[10] Wladimir Schamai, Philipp Helle, Peter Fritzson, and Christiaan J. J. Paredis. Virtual Verification of System Designs against System Requirements. In Models in Software Engineering - Workshops and Symposia at MODELS 2010, Oslo, Norway, October 2-8, 2010, Reports and Revised Selected Papers, pages 75-89, 2010.

[11] http://www.omgsysml.org.

[12] VDI. Design Methodology for Mechatronic Systems (VDI 2206). Technical report, VDI, 2004. 